\title{
Analysis of Human Binocular Fusional Area in Retinal Fovea
}

\author{
Damin QIN**, Mamoru TAKAMATSU**, Yoshio NAKASHIMA**, \\ Zojiro KATOH $^{* * *}$, Yasuaki FUKUTA ${ }^{* * * *}$
}

\begin{abstract}
In three-dimensional display systems, binocular disparities must be limited within a certain fusional area, called as "Panum's fusional area", otherwise too large disparity could cause double view or serious eye tiresome. In this study, a three-dimensional display device was employed with a constant stimuli method to measure the disparity limits of the Panum's fusional area in sixteen different directions from 0 degree to 360 degrees by a step of 22.5 degrees in the retinal fovea. The following results were obtained: (1) the horizontal disparity limit of binocular fusional area is about $32-40 \mathrm{~min}$ of arc and the vertical limit is about 19.2-25.6 min of arc. (2) The disparity limits of binocular fusional area are approximatively symmetrical about horizontal meridian. (3) The disparity limits are not symmetrical about vertical meridian, and the nasalward disparity limits are obviously larger than the temporalward disparity limits. (4) In the nasal side of retina, the disparity limits decrease in a monotonic fashion and in the temporal side, however, the disparity limits have no obvious difference. (Key word: Panum's fusional area, binocular disparity, three-dimensional display, retinal fovea)
\end{abstract}

\section{Introduction}

In recent years, the development of threedimensional display systems has been the subject of substantial research. Three-dimensional display systems would greatly enhance visualization of video and computer graphics, architectural plans, flight simulations, as well as education and personal entertainment. Among many types of three-dimensional display systems, autostereoscopic systems, such as parallax barrier designs, lenticular element designs and micro-polarizer designs, are widely used, because these systems do not require the observer to wear any device, such as glasses, to separate the left and right views, and instead send them directly to correct eye. In these three-dimensional display systems the human visual system perceives depth through binocular disparity cues. In other words, artificial binocular disparities are needed to be created in threedimensional display systems, so that observer could

\footnotetext{
*Received July 9, 2004; revised April 19, 2005

${ }^{* *}$ Faculty of Engineering, Toyama University

***Achi Mizuho College

${ }^{* * * *}$ Meijo University
}

obtain stereopsis perception. However unsuitable artificial disparities cause double view and serious eye tiresome. Therefore the artificial disparities should be limited within a certain binocular fusional area, which is known as "Panum's fusional area".

Studies about the Panum's fusional area have been highly researched topics all the time ${ }^{1-7)}$. The limits of the Panum's fusional area on horizontal and vertical meridian were frequently studied ${ }^{1 \sim 5)}$. However the limits of Panum's fusional area in the other oblique directions, such as 45, 135, 225 degrees etc., were seldom measured, except that Fender and Julesz 3) determined the limit of the Panum's fusional area at 45 degrees direction to horizontal meridian only in nasal side. Therefore, it is necessary to fully measure the disparity limits of Panum's fusional area in more directions. In this study, a micro-polarizer type three-dimensional display was employed to present the virtual experimental stimuli instead of the actual physical stimuli, used in the previous studies ${ }^{1-5)}$. Because of the convenience of controlling the threedimensional display device by a computer, we created a pair of virtual dichoptic stimulus targets on the 
three-dimensional display device to perceive a binocular view, and controlled some parameters of the stimuli, such as shape, size, position, color, etc. Therefore, it is possible to fully measure the limits of the binocular fusional area in any direction and in any position in retina.

The aim of the present study is to fully measure the disparity limits of Panum's fusional area in sixteen different directions from 0 degree to 360 degrees by a step of 22.5 degrees in retinal fovea, in addition to the horizontal and vertical meridian studied previously, so as to provide fundamental data for three-dimensional display systems to present more comfortable stereoscopic view.

\section{Method}

\section{2-1. Apparatus and stimuli}

In this experiment, a micro-polarizer type threedimensional display device (SANYO THD-10P3, display resolution $640 \times 480$, refresh rate $60 \mathrm{~Hz}$ ) was employed to present experimental stimuli dichoptically, i.e. one to each eye of the subject. As shown in Fig.1 (a), subject seated in front of the three-dimensional display device, was presented dichoptically a left eye view and a right eye view by the optical polarization action of the image splitter installed behind the LCD panel, hence perceived a binocular vision. A computer with a software system using Visual $\mathrm{C}++$, was used to control the three-dimensional display to show the experimental stimuli. The distance between the subject and the three-dimensional display device was $70 \mathrm{~cm}$ and a chin-rest and head-rest system was used in this experiment for fixing the head of subject against sliding.

The experimental stimuli consisted of a stationary white fixation dot and a white single line for each the left eye view and the right eye view, on a black background. As shown in Fig.1, (b) the fixation dot subtended a visual angle $48 \mathrm{~min}$ of arc, (c) the line target subtended a visual angle 48 min of arc in height and $3 \mathrm{~min}$ in width.

In this experiment, the investigation was made using a method of constant stimuli, with an exposure time of the stimulus target shorter than the latency period for eye movement in response to a convergence stimulus. This latency period has been found to be $140 \mathrm{~ms}$ by Ginsborg ${ }^{9}$, 150-200 ms by Westheimer and Mitchell $^{10)}$, and $160 \mathrm{~ms}$ by Rashbass and Westheimer ${ }^{11}$. Therefore, the exposure time of the stimuli adopted $140 \mathrm{~ms}$ in the present experiment.

\section{2-2. Subjects}

Data were obtained from five subjects aged between 22 and 28 years. All of them with normal visual acuities and having no oculomotor problem.

\section{2-3. Procedure}

After a subject was adapted to the dark about 45 min, a pair of fixation dots, which were respectively projected into the central fovea of left and right eye retinas, were presented for 3 seconds, afterward, the

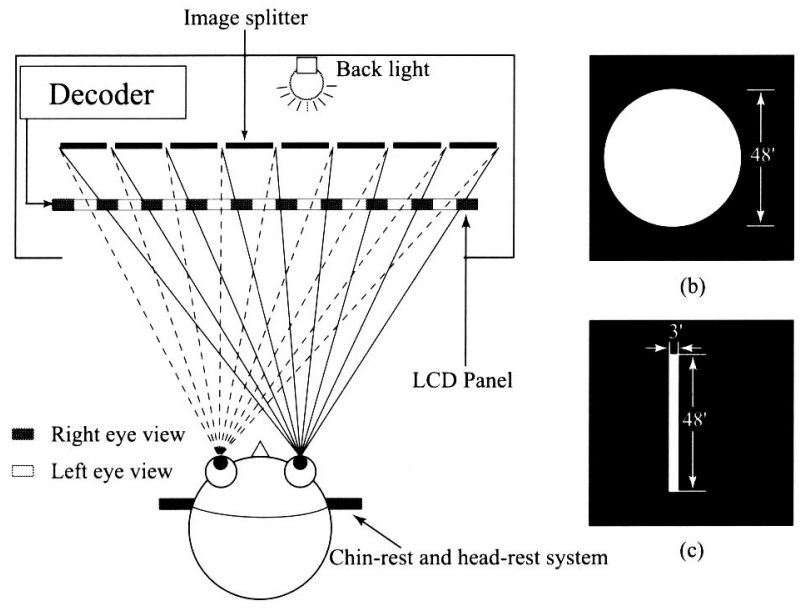

(a)

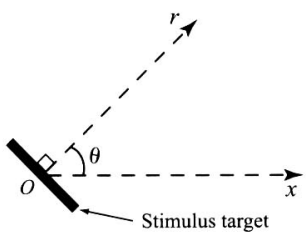

(d) Left eye view

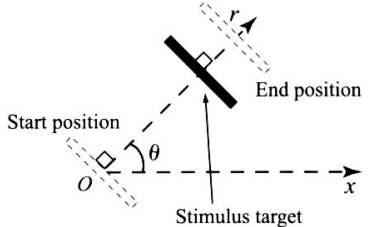

(e) Right eye view
Fig. 1 A schematic plan of three-dimensional display device (a), and experimental stimuli consisted of a white stationary fixation $\operatorname{dot}(\mathrm{b})$ and a white line target (c) for each eye on a black background. During a trial with a certain angle $\theta$, (d) the line target in the left eye view was always fixed in fovea, and (e) the line in the right eye view was changed from start position to end position in a random way. 


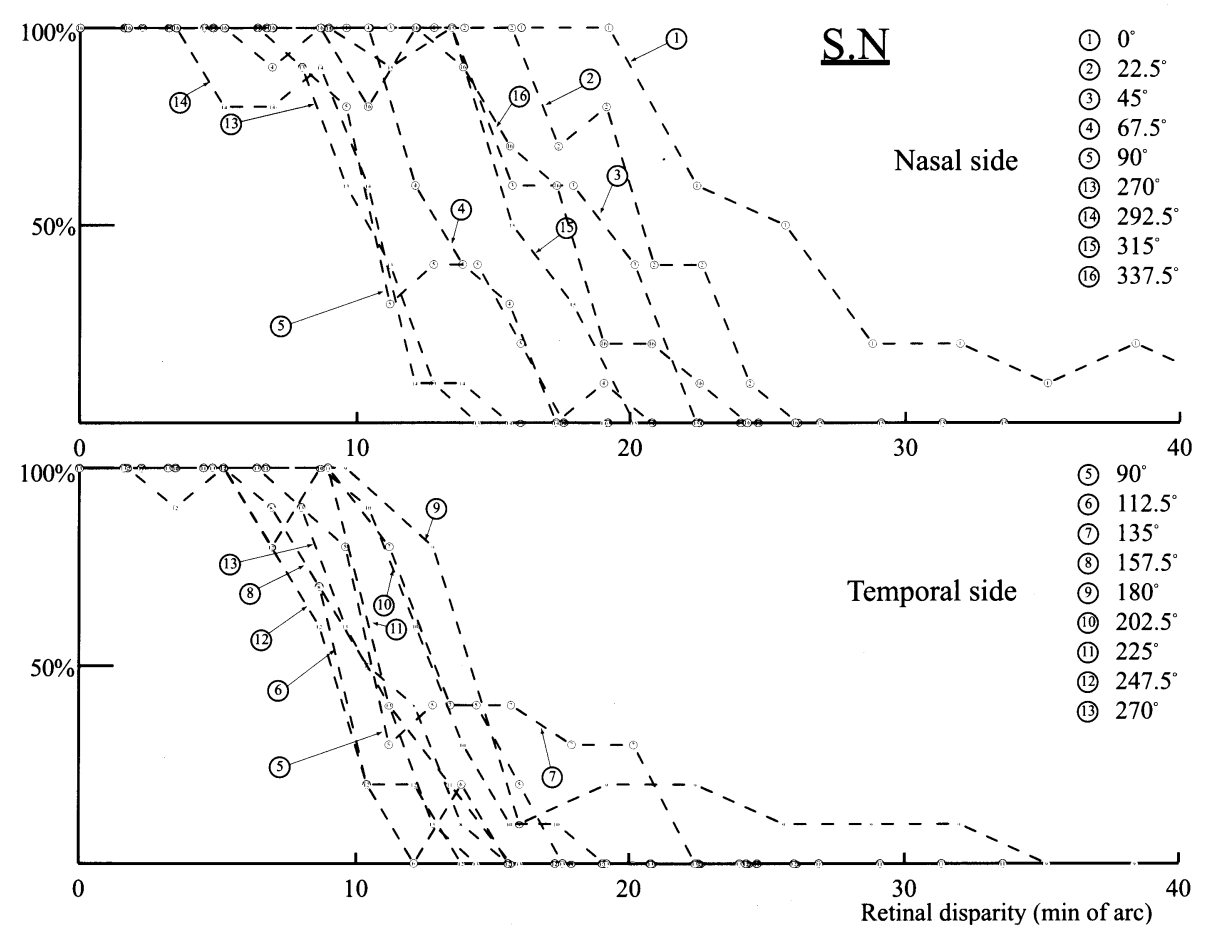

Fig. 2 The probability of fusion as function of retinal disparity (for subject S.N) in sixteen different directions.

fixation dots were turned off. At the same instant, the line stimulus targets for the left and right eye views simultaneously appeared, and lasted for $140 \mathrm{~ms}$. Then the subject was instructed to respond his or her perception was "single" or "double", as described in Tab.1, which was also used by Diner and Fender ${ }^{12)}$. In order to clearly express how to alter the position of the line stimulus targets, the polar coordinates were introduced into the present experiment, as shown in Fig.1 (d) and (e). The central point $o(0,0)$ in the left and right eye view respectively corresponded to the central fovea in the left and right retina. The $x$-axis corresponded to the horizontal meridian in the nasal side of retina. The experiment was repeated by altering the position of line stimulus target only in right retina view (Fig.1 (e)), along the radial coordinate $r$ with a certain angle $\theta$, in a random way among chosen values between start position and end position. While such alteration, the line stimulus target in the left eye view (Fig.1 (d)) was always kept in the central fovea of the left retina, until all positions within the chosen range were measured. The altering interval was 1 pixel in three-dimensional display (about $1.6 \mathrm{~min}$ of arc). In all experimental conditions, both line stimulus targets in left and right eye view were set to be
Tab. 1 Possible response in experiment.

\begin{tabular}{ccc}
\hline Category & Percept & Response \\
\hline A & Single & 1 \\
B & Probably single & 2 \\
C & Not sure & 3 \\
D & Probably double & 4 \\
E & Double & 5 \\
\hline
\end{tabular}

always perpendicular to the radial coordinate $r$.

In the present experiment, sixteen experimental conditions were obtained by varying the polar angle $\theta$ from 0 degree to 360 degrees by a step of 22.5 degrees. Each experimental condition was repeated 10 times for each subject. Moreover, only one experimental condition was done in one trial for each subject; the interval to next trial was at least one day.

The probability of fusion at each retinal disparity was calculated as follows:

$p_{d}=\frac{\text { Total number of reports in categories }(A+B)}{\text { Total number of reports in categories }(A+B+D+E)}$

\section{Results and discussion}

The results of the probability of fusion were obtained from five subjects. As a sample, the results only for subject S.N are shown in Fig.2. The results 


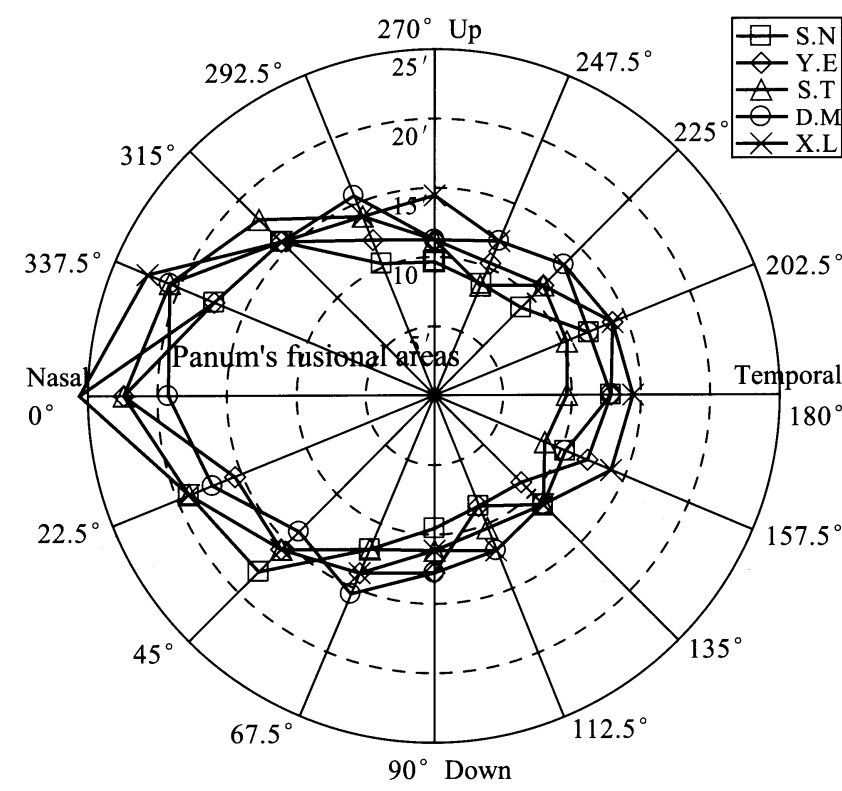

Fig. 3 Panum fusional areas in right eye retina for five subjects.

are divided into two parts of nasal side limits and temporal side limits, where also including the cases of 90 degrees and 270 degrees, though these cases belong to neither nasal nor temporal side. By using a 50 percent fusion criterion as the usual specifying threshold, the threshold disparities of the binocular single vision are obtained and shown in Fig.3 for all subjects. The center of Fig. 3 corresponds to the central fovea of the right retina. The areas surrounded by solid curves are Panum's fusional areas in fovea for five subjects. In other words, in the present experiment the line target in the left central fovea with the line target in the right retina, could always fuse into a binocular single vision, if and only if the line target in the right retina is fallen within the Panum's fusional area. It is noticed that in this study the directions of fusional area expressed in the Fig.3, such as temporal, nasal, up, down, is based on retina contrary to outside view.

As can be seen from Fig.3, the disparity limits in horizontal meridian, the total of nasalward and temporalward disparity limits (0 degree and 180 degrees), are about $32-40 \mathrm{~min}$ of arc. These values are rather different from some previous results ${ }^{1,2)}$. In fact, not all the previous results are strictly comparable, due to the differences in the experimental techniques, such as the size of the stimulus target, the exposure time of the target, the criterion of threshold and so on. For example, Palmer ${ }^{1)}$ quoted a figure of about $25 \mathrm{~min}$ with a vertical line subtended $45 \mathrm{~min}$ in height, about 15-20 min with a test spot subtended 1.5 min by a 83 percent criterion of threshold, the exposure time was $10 \mathrm{~ms}$. Mitchell ${ }^{2)}$ also determined this limit about 19 min with a vertical $42 \mathrm{~min}$ line, the fusion criterion was 50 percent and the exposure time was $120 \mathrm{~ms}$. We also learn that the vertical disparity limits are about 19.2-25.6 min of arc, containing upward limits (270 degrees) and downward limits (90 degrees), which are seen evidently smaller than horizontal disparity limits ( 0 degree and 180 degrees). This point has been reported in many previous experiments ${ }^{3,8}$.

Although Fender and Julesz ${ }^{3)}$ had never measured the limit of temporal side, they assumed the temporal side was symmetrical with nasal side about vertical meridian, so an ellipse as the form of Panum's fusional was suggested in their study. In the present experiment, as could be seen from Fig. 3 that the disparity limits are not symmetrical about vertical meridian, and nasalward limits are likely larger than temporalward limits. This phenomenon is common to all subjects and could be seen clearly by calculating the ratio of the nasalward limits to temporalward limits. For all subjects, the disparity limit ratios are over 1 . The minimum value of 1.2 was obtained for subject Y.E in the case of 67.5 degrees (nasal side) to 112.5 degrees (temporal side), and the maximum value of 2.3 was obtained for subject S.T in the case of 0 degree (nasal side) to 180 degrees (temporal side). Richards ${ }^{13)}$ also determined the uncrossed and crossed disparity limits, which correspond to the cases of 0 degree (nasalward) and 180 degrees (temporalward) in the present experiment (Fig.3). Richards changed the viewing distance from $\infty$ to $20 \mathrm{~cm}$, however we used a constant viewing distance of $70 \mathrm{~cm}$. If the viewing distance of $70 \mathrm{~cm}$ in the present experiment is applied to the experiment of Richards, we find that the uncrossed (nasalward) disparity limit is larger than the crossed (temporalward) disparity limit for all subjects in his experiment, which is same as our results.

The ratios of downward disparity limit to upward limit are also calculated to show that the values are about 1 , and it seems that the disparity limits are almost symmetrical about horizontal meridian. 
Fender and Julesz ${ }^{3)}$ examined the disparity limits at 45 degrees to the horizontal meridian in only nasal side with a moving stimuli target, consisting of nasaldownward and nasal-upward limits, which correspond to the cases of 45 degrees and 315 degrees in the present experiment (Fig.3). They revealed these disparity limits were situated between the vertical meridian limit (90 or 270 degrees) and horizontal meridian limit in the nasal side (0 degree). We also obtained similar results in our experiment; the disparity limits decrease monotonically with varying measurement direction from horizontal meridian in the nasal side (0 degree) to vertical meridian (90 or 270 degrees). However, as shown in Fig.3, the situation of the temporal side is very different from the nasal side. In the temporal side, the vertical disparity limit, either the upward or downward limit, is not minimum. Actually, there are slight differences of disparity limits in the temporal side of retina (from 90 degrees to 270 degrees), and the standard deviations of limits in the temporal side for five subjects are calculated as 1.5 min of arc for subject S.N, $1.75 \mathrm{~min}$ for subject Y.E, $1.07 \mathrm{~min}$ for subject S.T, $0.94 \mathrm{~min}$ for subject D.M, and $1.31 \mathrm{~min}$ for subject X.L.

In this experiment, we always held the fixation dot in the left retinal fovea while randomly varying the positions of line stimulus target in right eye view. The contrary condition never has been done. However we hypothesize the behavior of each of eye is identical.

The different fusion criterion for each subject is certainly one of the important causes affecting the apparent limits of binocular fusional area and individual variation. Furthermore, the resolution of the threedimensional display device $(640 \times 480)$ could also affect the precision measurements in disparity limit.

\section{Conclusions}

From the results, we suggest that, in threedimensional display systems, the horizontal disparity limits about $32-40 \mathrm{~min}$ and the vertical disparity limits about 19.2-25.6 min could not be exceeded, otherwise the observer could not obtain a single and comfortable view. In addition, the results reveal that human binocular fusional area in retinal fovea are almost symmetrical about the horizontal meridian. The disparity limits of nasal side and temporal side presented two independent behaviors. In temporal side of retina, the disparity limits have no difference from 90 degrees to 270 degrees. Nevertheless in the nasal side of retina, the disparity limits are found to decrease in a monotonic fashion as measurement direction varying from nasal horizontal meridian (0 degree) to vertical meridian (90 or 270 degrees). Moreover, this study clearly demonstrates that the disparity limits are not symmetrical about vertical meridian, and the nasalward limits are larger than the temporalward limits.

\section{References}

1) D. A. Palmer: Measurement of the horizontal extent of Panum's area by a method of constant stimuli, Optica Acta 8, 151-159, 1961.

2) D. E. Mitchell: Retinal disparity and diplopia, Vision Res., 441-451, 1966.

3) D. Fender and B. Julesz: Extension of Panum's fusional area in binocularly stabilized vision, J. Opt. Soc. Am., 57, 819-830, 1967.

4) T. P. Piantanida: Stereo hysteresis revisited, Vision Res., 26(3), 431-437, 1986.

5) D. B. Diner and D. H. Fender: Hysteresis in human binocular fusion: temporalward and nasalward ranges, J. Opt. Soc. Am., 4, 1814-1819, 1987.

6) S. L. Hwan and C. D. Allan : Fixation disparity and nonius bias, Vision Res., 39(3), 669-677, 1999.

7) M. T. Ukwade, et al.: Stereopsis is perturbed by vergence error, Vision Res., 43(2), 181-193, 2003.

8) Lyding: Über die netzhautdeckstellen, Klin. Mbl. AugenReilk, 102, 874, 1939.

9) B. L. Ginsborg: Small voluntary movements of the eye, Br. J. Ophthal, 37, 746-754, 1953.

10) G. Westheimer and G. Mitchell: Eye movement responses to convergence stimuli, Arcd Ophthal, 55, 848-856, 1956.

11) C. Rashbass and G. Westheimer: Disjunctive eye movements, J. Physiol.,159, 339-360, 1961.

12) D. B. Diner and D. H. Fender: Dependence of Panum's fusional area on local retinal stimulation, J. Opt. Soc. Am., 5, 1163-1169, 1988.

13) W. Richards: Independence of Panum's near and far limits, Amer. J. Opt. \& Arch. Amer. Acad. Opt., 48, 103-109, 1971. 